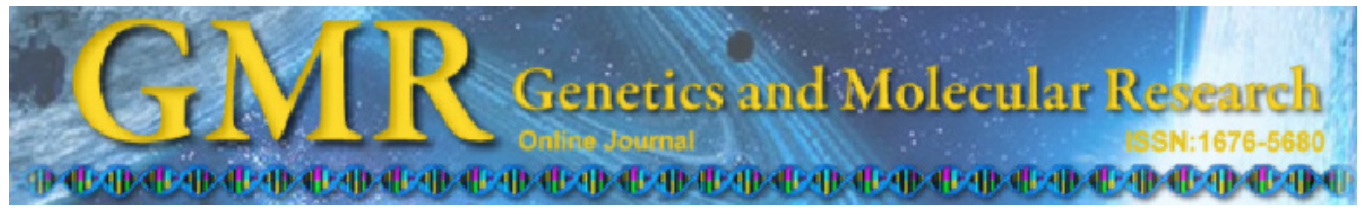

\title{
Plasma chemerin level in metabolic syndrome
}

\author{
D. Wang, G.-Y. Yuan, X.-Z. Wang, J. Jia, L.-L. Di, L. Yang, X. Chen, \\ F.-F. Qian and J.-J. Chen
}

Department of Endocrinology and Metabolism,

Affiliated Hospital of Jiangsu University, Jiangsu, China

Corresponding author: G.-Y. Yuan

E-mail: dongdonghao_wd@163.com

Genet. Mol. Res. 12 (4): 5986-5991 (2013)

Received November 11, 2012

Accepted August 20, 2013

Published November 26, 2013

DOI http://dx.doi.org/10.4238/2013.November.26.8

\begin{abstract}
To investigate the chemerin level in the Chinese Han population with metabolic syndrome and its relationship with each metabolic syndrome component [body mass index (BMI), blood pressure, blood lipids, and blood glucose], we selected 30 patients with metabolic syndrome and 30 healthy control subjects. The chemerin level was measured by enzyme immunoassay in these 2 groups. The subjects' weight, blood pressure, BMI, waist circumference, fasting blood glucose, fasting insulin, lipids, and glycated hemoglobin were simultaneously detected. The $t$-test, correlation analysis, and multiple regression analysis were used to perform statistical analysis. We found that plasma chemerin level was higher in the metabolic syndrome group than that in the control group $(97.61 \pm 6.49 v s 70.26 \pm 6.97, \mathrm{t}=15.73, \mathrm{P}<0.05)$. The plasma chemerin level was positively correlated with systolic blood pressure, waist circumference, BMI, waist-to-hip ratio, fasting blood glucose, fasting insulin, and glycated hemoglobin $(\mathrm{r}=0.548,0.442,0.359$, $0.556,0.613,0.581$, and 0.572 , respectively; all $\mathrm{P}<0.05$ ). However, it was negatively correlated with high-density lipoprotein cholesterol $(\mathrm{r}=-0.378, \mathrm{P}<0.05)$. Therefore, we concluded that plasma chemerin level was correlated with obesity, blood pressure, and high-density lipoprotein cholesterol, suggesting that it may play a role in the
\end{abstract}


pathogenesis of metabolic syndrome.

Key words: Metabolic syndrome; Chemerin

\section{INTRODUCTION}

Metabolic syndrome (MS) is a series of biochemical clinical metabolic abnormalities mainly caused by insulin resistance (IR). It includes obesity, diabetes or impaired glucose tolerance, dyslipidemia, hypertension, microalbuminuria, and other major risk factors and is, in essence, the aggregation of multiple metabolic risk factors for cardiovascular disease (Eckel et al., 2005). With obesity increasing globally, the incidence of MS continues to grow. Chemerin is a recently discovered adipokine secreted by fat cells. It is highly expressed in adipose tissue. Recent studies have shown that endocrine dysfunction of adipose tissue is an important reason for IR, as chemerin is associated with obesity, IR, and metabolic syndrome (Ailhaud, 2006; Bozaoglu et al., 2007). One study in China has found several key chemerin indicators of MS such as IR in the Uyghur population (Guan et al., 2010); however, similar studies are rare in the Chinese Han population. This study compared the plasma chemerin levels in healthy subjects and MS patients in the Han population to explore the relationship of chemerin with MS.

\section{MATERIAL AND METHODS}

\section{Subjects}

Subjects were selected from a 2008 survey of the prevalence of MS in Zhenjiang (China) and from among patients visiting the clinic, inpatient, and health examination departments of the Affiliated Hospital of Jiangsu University from 2008 to 2011. All subjects were Chinese Han. The control group included 30 healthy people - 18 men and 12 women - with a mean age of 51 \pm 9 years. These subjects had no family history of diabetes, high cholesterol, or hypertension. Two-hour post-load plasma glucose was tested with 75 g glucose (or 82.5 g glucose containing 1 molecule of water) to exclude diabetes or impaired glucose tolerance. Subjects lacked abdominal obesity (male waist circumference $<90 \mathrm{~cm}$, females $<80 \mathrm{~cm}$ ) and abnormalities of blood lipids and blood pressure. None of the subjects had received antihypertensive, lipid, or glycemic control medications within a month of assessment. Thirty MS patients, consisting of 16 men and 14 women with a mean age of $49 \pm 8$ years, were also included in the study. The diagnosis of MS was based on the global consensus of MS according to the 2005 International Diabetes Federation (Song and $\mathrm{Ji}, 2005$ ). Patients must have central obesity (Chinese standard: male waist circumference $\geq 90 \mathrm{~cm}$, female waist circumference $\geq 80 \mathrm{~cm}$ ) and any 2 of the following 4 factors to make the diagnosis: 1$)$ increasing triglyceride $(\mathrm{TG})$ levels $(\mathrm{TG}>1.7 \mathrm{mM})$ or receiving special treatment for this lipid abnormality, 2) decreasing high-density lipoprotein cholesterol (HDL-C; $<1.03 \mathrm{mM}$ for men and $<1.29 \mathrm{mM}$ for women) or receiving special treatment for this lipid abnormality, 3 ) increasing fasting plasma glucose (FPG; $\geq 5.6 \mathrm{mM}$ ) or having been diagnosed with type II diabetes, 4) high blood pressure (systolic blood pressure $\geq 130 \mathrm{mmHg}$ or diastolic blood pressure $\geq 85 \mathrm{mmHg}$ ) or having been diagnosed with hypertension and treated. Subjects with thyroid dysfunction, liver and kidney dysfunction, and acute inflammation and trauma were excluded. The study was approved by the hospital ethics committee and consent was given by all subjects. 


\section{Specimen collection}

Blood specimens of fasting glucose and glucose $2 \mathrm{~h}$ after the test were collected after fasting 10-14 h overnight. After the plasma was centrifuged, the blood samples were divided in half. One half was used to test the relevant biochemical markers immediately, and the other was added to aprotinin and stored at $-70^{\circ} \mathrm{C}$ for further chemerin testing.

\section{Clinical indicator observation}

Height, weight (wearing underwear only), waist circumference (at the subcostal level and midpoint of the anterior superior iliac spine line), hip circumference (maximum extension area of the buttocks), blood pressure (interval of $2 \mathrm{~min}$; blood pressure was measured twice and recorded as the average), body mass index $\left(\mathrm{BMI}=\right.$ body weight $\left./ \mathrm{height}^{2} ; \mathrm{kg} / \mathrm{m}^{2}\right)$, and waist $/ \mathrm{hip}$ ratio $(\mathrm{WHR}=$ waist circumference/hip circumference) of the subjects were measured.

\section{Biochemical indicators}

FPG, FINS, TG, total cholesterol (CHOL), HDL-C, low-density lipoprotein cholesterol (LDL-C), glycated hemoglobin (HbA1c), and plasma chemerin levels were detected using the venous blood collected as described above after 10-14 h of fasting. The glucose and lipids were detected using an Olympus AU2700 automatic biochemical analyzer (Olympus, Japan). $\mathrm{HbA1c}$ was measured using a high-pressure liquid method. The insulin level was measured through radioimmunoassay determination of the plasma. Chemerin was detected using an enzyme immunoassay kit (linear range, $0.032-2.200 \mathrm{ng} / \mathrm{mL}$, with an intra-difference of $<4.9 \%$; R\&D Systems, USA).

\section{Statistical analysis}

SPSS11.5 (SPSS, Chicago, IL, USA) statistical analysis software was used. All continuous variables are reported as means \pm standard deviation. A $t$-test was used to compare significance among groups. Non-normally distributed variables were converted into normally distributed variables for analysis. The relationship between chemerin and other variables was detected using linear correlation analysis and multiple regression analysis, with a $\mathrm{P}$ value of $<0.05$ indicating statistically significant difference.

\section{RESULTS}

\section{General clinical data comparison}

The gender ratio and ages of the subjects in the 2 groups were not significantly different. Body weight, waist circumference, BMI, and WHR in the MS group were greater than those in the control group $(71.9 \pm 8.87$ and $63.33 \pm 7.92, \mathrm{t}=3.95 ; 93.23 \pm 6.05$ and $80.40 \pm$ $7.71, \mathrm{t}=7.17 ; 25.9 \pm 1.94$ and $22.04 \pm 1.32, \mathrm{t}=9.01 ; 0.92 \pm 0.02$ and $0.89 \pm 0.01, \mathrm{t}=7.35$, respectively; all $\mathrm{P}<0.05$ ). Systolic and diastolic blood pressures in the MS group were also higher than those in the control group $(136.17 \pm 8.43$ and $120.33 \pm 5.59, \mathrm{t}=8.58 ; 79.97 \pm 7.39$ and $73.27 \pm 6.02, \mathrm{t}=3.85$; all $\mathrm{P}<0.05$; Table 1$)$. 
Table 1. Comparison of the clinical indicators of the two groups.

\begin{tabular}{lccccccccc}
\hline Group & Case $(\mathrm{n})$ & Age (years) & Height $(\mathrm{cm})$ & Weight $(\mathrm{kg})$ & BMI $\left(\mathrm{kg} / \mathrm{m}^{2}\right)$ & WC $(\mathrm{cm})$ & WHR & SBP $(\mathrm{mmHg})$ & DBP $(\mathrm{mmHg})$ \\
\hline Control Group & 30 & $51.37 \pm 9.27$ & $169.11 \pm 6.31$ & $63.33 \pm 7.92$ & $22.04 \pm 1.32$ & $80.40 \pm 7.71$ & $0.89 \pm 0.01$ & $120.33 \pm 5.59$ & $73.27 \pm 6.02$ \\
MS Group & 30 & $49.03 \pm 8.34$ & $166.30 \pm 5.72$ & $71.90 \pm 8.87$ & $25.90 \pm 1.94$ & $93.23 \pm 6.05$ & $0.92 \pm 0.02$ & $136.17 \pm 8.43$ & $79.97 \pm 7.39$ \\
t value & 1.03 & 1.81 & 3.95 & 9.01 & 7.17 & 7.35 & 8.58 & 3.85 \\
P & $>0.05$ & $>0.05$ & $<0.05$ & $<0.05$ & $<0.05$ & $<0.05$ & $<0.05$ & $<0.05$ \\
\hline
\end{tabular}

$1 \mathrm{~mm} \mathrm{Hg}=0.133 \mathrm{kPa}$. BMI = body mass index, $\mathrm{WC}=$ waist circumference, $\mathrm{WHR}=$ waist $/$ hip ratio, $\mathrm{SBP}=$ systolic blood pressure, $\mathrm{DBP}=$ diastolic blood pressure.

Table 2. Comparison of the plasma chemerin level and the other indicators of the two groups.

\begin{tabular}{lcccccccc}
\hline Group & FPG $(\mathrm{mM})$ & FINS $(\mathrm{mIU} / \mathrm{L})$ & HbA1c $(\%)$ & TG $(\mathrm{mM})$ & CHOL $(\mathrm{mM})$ & LDL-C $(\mathrm{mM})$ & HDL-C $(\mathrm{mM})$ & Chemerin $(\mathrm{ng} / \mathrm{mL})$ \\
\hline Control Group & $4.78 \pm 0.42$ & $7.15 \pm 1.19$ & $5.41 \pm 0.49$ & $0.14 \pm 0.08$ & $4.29 \pm 0.61$ & $3.32 \pm 0.47$ & $1.41 \pm 0.18$ & $70.26 \pm 6.97$ \\
MS Group & $6.75 \pm 1.23$ & $13.44 \pm 2.25$ & $6.78 \pm 0.61$ & $0.36 \pm 0.14$ & $4.34 \pm 0.62$ & $3.46 \pm 0.41$ & $1.14 \pm 0.12$ & $97.61 \pm 6.49$ \\
t value & 8.30 & 13.53 & 9.59 & 7.67 & 0.32 & 1.23 & 6.84 & 15.73 \\
P & $<0.05$ & $<0.05$ & $<0.05$ & $<0.05$ & $>0.05$ & $>0.05$ & $<0.05$ & $<0.05$ \\
\hline
\end{tabular}

$\mathrm{FPG}=$ Fasting plasma glucose, $\mathrm{FINS}=$ fasting insulin, $\mathrm{TG}=$ triglyceride, $\mathrm{CHOL}=$ total cholesterol, $\mathrm{LDL}-\mathrm{C}=$ lowdensity lipoprotein cholesterol, HDL-C = high-density lipoprotein cholesterol.

\section{Comparison of clinical and biochemical data}

FPG, FINS, TG, CHOL, LDL-C, and HbA1c in the MS group were higher than those in the control group $(6.75 \pm 1.23$ and $4.78 \pm 0.42, \mathrm{t}=8.30, \mathrm{P}<0.05 ; 13.44 \pm 2.25$ and $7.15 \pm 1.19$, $\mathrm{t}=13.53, \mathrm{P}<0.05 ; 0.36 \pm 0.14$ and $0.14 \pm 0.08, \mathrm{t}=7.67, \mathrm{P}<0.05 ; 4.34 \pm 0.62$ and $4.29 \pm 0.61$, $\mathrm{t}=0.32, \mathrm{P}>0.05 ; 3.46 \pm 0.41$ and $3.32 \pm 0.47, \mathrm{t}=1.23, \mathrm{P}>0.05 ; 6.78 \pm 0.61$ and $5.41 \pm 0.49, \mathrm{t}$ $=9.59, \mathrm{P}<0.05$, respectively). HDL-C was lower in the MS group than that in the control group $(1.14 \pm 0.12$ and $1.41 \pm 0.18, \mathrm{t}=6.84, \mathrm{P}<0.05)$, and chemerin level was higher in the MS group than that in the control group $(97.61 \pm 6.49$ and $70.26 \pm 6.97, \mathrm{t}=15.73, \mathrm{P}<0.05$; Table 2$)$.

\section{Correlation analysis}

The correlation coefficient $r$ values of the plasma chemerin level and body weight, BMI, waist circumference, WHR, systolic blood pressure, diastolic blood pressure, TG, CHOL, LDL-C, HDL-C, FPG, FINS, and HbA1c were 0.104, 0.389, 0.442, 0.556, 0.548, 0.252, 0.242, $0.361,0.223,-0.378,0.613,0.581$, and 0.572 , respectively. Systolic blood pressure, waist circumference, BMI, WHR, FBG, FINS, and HbAlc were positively correlated with the plasma chemerin level $(\mathrm{r}=0.548,0.442,0.389,0.556,0.613,0.581,0.572$, respectively; $\mathrm{P}<0.05)$. HDL-C was negatively correlated with plasma chemerin level $(r=-0.378, \mathrm{P}<0.05)$. Chemerin was considered the dependent variable, and the various components in MS (e.g.; waist circumference, WHR, FPG, TG, HDL-C, systolic blood pressure, and diastolic blood pressure) were considered the independent variables. Multiple regression analysis was performed, and the systolic blood pressure, BMI, and WHR were placed into the equation $(\mathrm{P}<0.05)$.

\section{DISCUSSION}

Wittamer et al. (2003) isolated the ligands of the G protein-coupled receptor chemR23 from the ascites of the patients with ovarian cancer and identified a chemoattractant protein that they called chemerin. In inflammatory states, it can gather macrophages and immature dendritic 
cells carrying chemR23 to sites of inflammation to regulate local inflammatory reactions. Goralski et al. (2007) have used real-time quantitative polymerase chain reaction to identify chemerin as an adipokine and found that it is expressed in liver, spleen, pancreas, kidney, and ovary. Takahashi et al. (2008) have studied 3T3-L1 adipocytes cells and found that chemerin enhances insulin signaling, increases insulin-stimulated glucose transport, and regulates insulin sensitivity to adipose tissue. Chemerin is a new adipokine that regulates fat formation and metabolism and affects the metabolism of fat cell differentiation and adipose tissue inflammatory response through the paracrine and autocrine systems. Sell et al. (2009) examined human skeletal muscle cells and found that chemerin induces IR. Adipose tissue chemerin secretion was higher in obese patients than that in non-obese control subjects, which proved that chemerin secretion is associated with fat volume. Other studies have suggested that the secretion of adipocytokines is closely related to obesity, IR, and metabolic syndrome (Ailhaud, 2006; Bozaoglu et al., 2007; Ding and Fan, 2009). Chemerin may be closely related to MS as an adipokine.

The 3rd report of the United States Cholesterol Education Program Adult Treatment Panel (Ford and Giles, 2003) stated that the prevalence of MS was $23.9 \%$ in the United States. In China, Yang et al. (2012) have recently published data showing that the incidence of overweight and obesity has doubled compared with that in 2002, and the total population exceeded 10 million. However, the etiology and pathogenesis of MS has not been elucidated. It occurs because of complex interactions between genetic and environmental factors. IR is generally accepted as a central component of MS, and obesity - particularly central obesity - is closely related to IR. According to International Diabetes Federation (IDF) guidelines, abdominal circumference increase and central obesity are a prerequisite of MS. Fat distribution affects the development of MS as well. Obesity is now considered a chronic low-level inflammation status, and the size and number of fat cells directly affects the pathological changes of obesity. Adipose tissue increases abdominal obesity, and in turn chemerin secretion increases. This expression imbalance may lead to the abnormal metabolism and differentiation of fat tissue and result in the pathological changes of obesity.

Our study found a significant difference in the weight, waist circumference, WHR, and $\mathrm{BMI}$ in the MS group compared with that in the control group. Chemerin level was significantly higher in the MS group than that in the control group, and plasma chemerin level was positively correlated to waist circumference, systolic blood pressure, BMI, WHR, FPG, FINS, and HbA1c. A study by Ailhaud (2006) has reported that plasma chemerin level is significantly higher in individuals with a BMI of $>30 \mathrm{~kg} / \mathrm{m}^{2}$ than that in individuals with a BMI of $>25 \mathrm{~kg} / \mathrm{m}^{2}$. BMI reflects the degree of the systemic body fat and obesity. Waist circumference is an indicator of local fat and body fat distribution and better reflects the actual status of central obesity: the larger the waist circumference, the more significant the central obesity. Stejskal et al. (2008) have found that chemerin level is correlated with waist circumference and BMI in whites after adjustment for age, gender, blood pressure, and blood lipids. Our results are consistent with the findings of these studies, showing that chemerin is correlated with MS characterized by central obesity. Central obesity may be among the reasons for elevated chemerin.

We observed that plasma chemerin was positively correlated with systolic blood pressure. Other studies have reported that chemerin is related to weight and blood pressure after adjustment for age and gender in subjects with normal glucose tolerance (Wittamer et al., 2003; Ailhaud, 2006). Chemerin combines with chemerin receptors on the cell membrane to inhibit adenylate cyclase activity and cyclic adenosine monophosphate aggregation. This process induces the release of calcium ions, increasing the concentration of intracellular 
calcium and causing the vasoconstriction that leads to high blood pressure. Chemerin may be a new factor in blood pressure regulation.

In addition, we found that chemerin level was negatively correlated with HDL-C level. HDLs provide a vascular protective effect by reducing the damage of vascular lesions, by lowering high blood pressure caused by vasospasm and vascular wall cell proliferation, for example. Our study also revealed that chemerin level and high blood pressure had an important relationship. Our findings differed from those of Guan et al. (2010) in Uyghur patients with MS. The reasons for the differences may be variations in genetic background, lifestyle habits, research protocols, and our limited sample size. MS is a combination syndrome with multiple dysfunctions. Our control subjects had an uncomplicated medical status without abnormalities in blood lipids, blood glucose, and blood pressure. Whether single-component or multiplecomponent abnormalities lead to changes in chemerin level remains to be studied further.

In summary, the correlation of plasma chemerin level and obesity, blood pressure, and HDL-C suggested that the recently described fat factor, chemerin, may play a role in the pathogenesis of MS. In-depth study of the biological function of chemerin, its role in fat differentiation, and its significance in the causality and development of MS will provide new ideas for predicting the development of MS and finding targets for drug therapy.

\section{ACKNOWLEDGMENTS}

Research supported by the Science and Technology Projects of Health Department of Jiangsu Province (\#H201247); the Social Development and Technologic Support Project of Zhenjiang (\#FZ2012030, \#SH2013035) and the Clinical Medicine Science and Technology Development Fund of Jiangsu University (\#JLY20120150).

\section{REFERENCES}

Ailhaud G (2006). Adipose tissue as a secretory organ: from adipogenesis to the metabolic syndrome. C R Biol. 329 : 570-577.

Bozaoglu K, Bolton K, McMillan J, Zimmet P, et al. (2007). Chemerin is a novel adipokine associated with obesity and metabolic syndrome. Endocrinology 148: 4687-4694.

Ding XD and Fan JG (2009). Hepatic insulin resistance mechanisms and consequences. Chin. J. Diabetes 1: 297-301.

Eckel RH, Grundy SM and Zimmet PZ (2005). The metabolic syndrome. Lancet 365: 1415-1428.

Ford ES and Giles WH (2003). A comparison of the prevalence of the metabolic syndrome using two proposed definitions. Diabetes Care 26: 575-581.

Goralski KB, McCarthy TC, Hanniman EA, Zabel BA, et al. (2007). Chemerin, a novel adipokine that regulates adipogenesis and adipocyte metabolism. J. Biol. Chem. 282: 28175-28188.

Guan YQ, Zhao XX and Sun LL (2010). The uighur metabolic syndrome patients of chemerin expression of and related factors. Chin. J. Hypertens. 18: 733-738.

Sell H, Laurencikiene J, Taube A, Eckardt K, et al. (2009). Chemerin is a novel adipocyte-derived factor inducing insulin resistance in primary human skeletal muscle cells. Diabetes 58: 2731-2740.

Song XX and Ji LN (2005). International Diabetes Federation global consensus on the definition of the metabolic syndrome. Chin. J. Diabetes 13: 178-180.

Stejskal D, Karpisek M, Hanulova Z and Svestak M (2008). Chemerin is an independent marker of the metabolic syndrome in a Caucasian population--a pilot study. Biomed. Pap. Med. Fac. Univ. Palacky. Olomouc. Czech Repub. 152: 217-221.

Takahashi M, Takahashi Y, Takahashi K, Zolotaryov FN, et al. (2008). Chemerin enhances insulin signaling and potentiates insulin-stimulated glucose uptake in 3T3-L1 adipocytes. FEBS Lett. 582: 573-578.

Wittamer V, Franssen JD, Vulcano M, Mirjolet JF, et al. (2003). Specific recruitment of antigen-presenting cells by chemerin, a novel processed ligand from human inflammatory fluids. J. Exp. Med. 198: 977-985.

Yang ZJ, Liu J, Ge JP, Chen L, et al. (2012). Prevalence of cardiovascular disease risk factor in the Chinese population: the 2007-2008 China National Diabetes and Metabolic Disorders Study. Eur. Heart J. 33: 213-220. 